RASĀYAN J. Chem.

Vol. 13 | No. 3 |1644-1652 | July - September | 2020

ISSN: 0974-1496 | e-ISSN: 0976-0083 | CODEN: RJCABP

RJC

http://www.rasayanjournal.com

http://www.rasayanjournal.co.in

\title{
STRATEGIES AND CHARACTERIZATION METHODS IN THE PREPARATION OF POLYMERIC SURFACTANTS FOR VARIOUS APPLICATIONS
}

\author{
Neeta Singh ${ }^{1, *}$, P.K.S. Yadav ${ }^{2}$ and S.K. Gupta ${ }^{1}$ \\ ${ }^{1}$ Department of Chemical Engineering, Harcourt Butler Technical University, \\ Kanpur, U.P., 208002, India \\ ${ }^{2}$ Department of Oil Technology, Harcourt Butler Technical University, \\ Kanpur, U.P., 208002, India \\ *E-mail: neetasinghrana1410@gmail.com
}

\begin{abstract}
The polymeric surfactants are gaining the attention of researchers and scientists because of their enhanced surfaceactive properties. In this review, an effort is made to highlight the application of polymeric surfactants and discuss the methods used for their synthesis from different types of feedstocks. The physicochemical properties of polymeric surfactants are superior to conventional surfactants with excellent surface-active features. The development of analytical methods for the characterization of polymers with complex macromolecular structural architectures is essential for the synthesis of polymeric surfactants. Emphasis is laid upon the discussion on the intermolecular micellar aggregates formed as a result of interactions between the polymer backbone and the amphiphilic micelles along with the impact of controlled polymerization to enhance the features of the polymeric surfactants in the detergent industry. An effort to comprehensively analyze the methods deployed for the synthesis of polymeric surfactants concerning their characteristic features that are unique to polymeric surfactants which enable them to be extensively used in the detergent industry is done. These discussions will help in understanding and designing polymeric surfactants for specific applications in the detergent industry.
\end{abstract}

Keywords: Amphiphilic micelles, Evaluation, Polymeric Surfactants, Polymerization.

(C) RASĀYAN. All rights reserved

\section{INTRODUCTION}

Polymeric surfactants have recently become popular in the detergent industry due to their surface-active properties. Commercially available detergents solubilize many chemical species by splitting molecular aggregates and by unfolding the tertiary structure of proteins. Polymeric surfactants are formulated such that they possess detergent-like association properties and are materials generally used to promote the removal of substances from the surface of fabrics and to disperse and stabilize substances in a bulk matrix. Polymeric surfactants can also influence the emulsification process that is essential for the functioning of any detergent ${ }^{1}$. Like detergents, polymeric surfactants can also be used in diluted quantities, but they still retain their cleaning properties. In the aqueous bulk phase, most of the detergents form surface aggregates; hence they are rightly called surfactants. Surfactants usually reduce the surface tension of an aqueous solution because they work efficiently at a liquid-air interface ${ }^{2}$. The polymerization of such surface-active materials enhances its hydrophilic-lipophilic balance, which in turn increases the dynamics of surfactant adsorption ${ }^{3}$. This acquired characteristic increases its foamability and produces better steric interactions on the absorption layers in a bulk solution. The principal function of these surfactants is to seamlessly modify the interface between two phases and promote the dispersion of the substances. The amphiphilic character of the surfactants effectively reduces the interfacial tension between water and any other material involved with $\mathrm{it}^{4}$. The association formed as a result of the supramolecular association between a polymer and a surfactant results in the formation of various structures such as molecular aggregates, coacervates, micellelike molecules, precipitates and hydrogels ${ }^{5}$. Some of the most studied polymer-surfactant mixtures are neutral hydrophilic polymers, copolymers, mixtures of surfactants and proteins, hydrophobically modified

Rasayan J. Chem., 13(3), 1644-1652(2020)

http://dx.doi.org/10.31788/RJC.2020.1335387

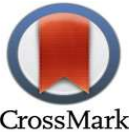


RASĀYAN J. Chem.

Vol. 13 | No. 3 |1644-1652| July - September | 2020

polymers and ionic surfactants ${ }^{6}$. Depending on the amphiphilic copolymer architecture and on solution parameters, the different micellar systems can be obtained (Fig.-1).

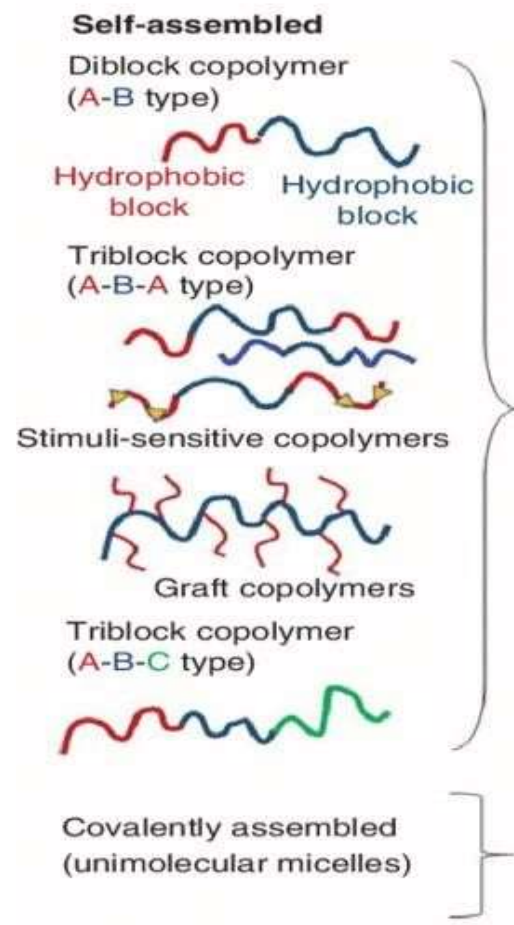

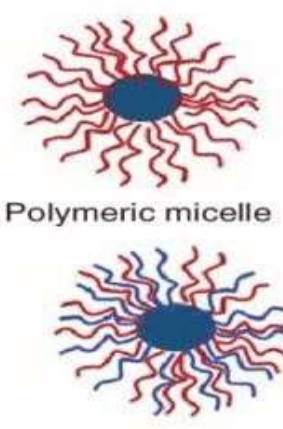

Mixed micelle

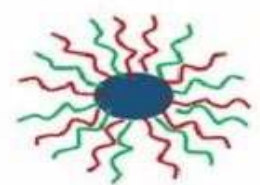

Polymeric micelle

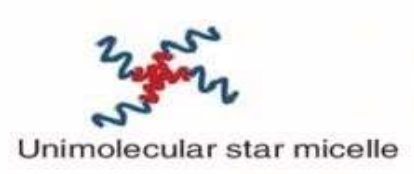

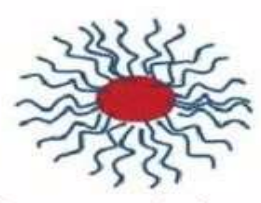

Reverse micelle

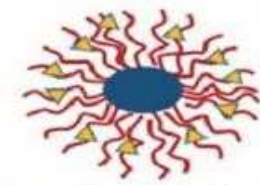

Sensitive micelle

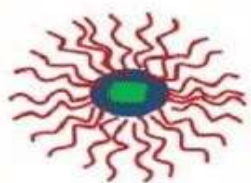

Multicompartment micelle

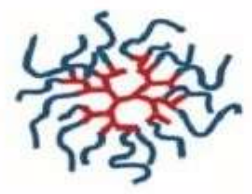

Unimolecular dentritic micelle

Fig.-1: Types of Polymeric micelle Formation Depending on the Copolymer Architecture and the Intermolecular Forces (Source: Simões, Figueiras, Veiga, Concheiro \& Alvarez-Lorenzo, 2014)

The association between the polymers and the surfactants is dependent on many variables such as the conformational compatibility, rigidity of the polymer or the associated protein, the type of surfactants used, the charge of both the molecules, the hydrophilic-lipophilic balance of the newly formed compound, $\mathrm{pH}$ and ionic strength, charge density, the temperature at which bond formation was facilitated and the formation of stable bonds also stabilize the polymeric molecules and improve its properties ${ }^{7}$ supramolecular molecular aggregates. Electrostatic bonding, Van der Waals forces, and hydrogen). Thus intricate balance is maintained for the formulation of polymeric surfactants and both the attractive and repulsive interactions must be stabilized depending on the chemical nature of the polymer and the surfactant used. The structural conformity is maintained by the release of counterions that strongly influence the entropy of the polymerization process. ${ }^{8}$ Based on their intermolecular and intramolecular forces surfactants can be classified as non-ionic, anionic, cationic and amphoteric surfactants. Non-ionic surfactants possess hydrophilic moieties such as polyoxyethylene, polyoxypropylene, alkanolamides, or esters. The surfactants with an anionic group like a sulfate molecule, sulfonate and a carboxylate are referred to as anionic surfactants. The surfactants possessing both anionic groups and cationic groups are called amphoteric surfactants or zwitterionic surfactants. ${ }^{9}$ The interactions between the polymer and the surfactants result in the formation of an aggregate that can efficiently drive the reduction of repulsion forces inside the micelle. This feature of the polymeric surfactant makes it extremely favorable for application in the detergent industry. Customized polymeric surfactants possess homopolymers or heteropolymers that assist in cleaning as they act as dispersants for inorganic salts and provide alkalinity. These polymeric surfactants inhibit the formation of inorganic crystal formations on fabrics. ${ }^{4}$ The surface absorption property of these polymeric surfactants is gaining an excellent commercial market in the detergent industry. Polymeric surfactants are products that can be tailor-made to be environmentally friendly and commercially useful. The chemical behavior of these polymeric surfactants is being explored to understand their critical aggregation concentrations to alter the size of the complexes to maintain stability and increase solubility. 
RASĀYAN J. Chem.

Vol. 13 | No. 3 |1644-1652| July - September | 2020

Synthetic polymers can also be modified such that the functionality can be amplified and the degradation can be reduced. ${ }^{4}$ Mass production of these polymeric molecules has the potential to replace conventionally used surfactants in the detergent industry. This review paper will give a brief account of the development strategies, classification and characterization and the potential applications of polymeric surfactants in the detergent industry. The main purposes of this review ${ }^{1}$ are to provide an overview of the methods of synthesis of polymeric surfactants, ${ }^{2}$ to analyze the characteristics of polymeric surfactants that are ideal for the detergent industry and ${ }^{3}$ to summarize the applications of polymeric surfactants.

\section{Classification of Polymeric Surfactants}

Polymeric surfactants can be classified based on the distribution of hydrophobic and hydrophilic moieties in the complex polymeric chain. Large complex macromolecules with intricately conjugated amphiphilic monomers are classified as polysoaps. These molecules contain surfactant like subgroups within the polymeric repeating unit. Homopolymers and heteropolymers, as well as copolymers of hydrophilic and hydrophobic monomers, are arranged in an alternating manner. Some polymeric surfactants are arranged in a linear neat pattern of monomers with amphiphilic moieties embedded systematically ${ }^{10}$, called macro surfactants (Fig.-2). Block copolymers with amphiphilic linear segregation between hydrophilic and hydrophobic groups are also included in the macro surfactant category of polymeric surfactants. Distinct intramolecular and intermolecular association of copolymers forms complex multiblock architectures with a tendency to form stable aggregate formation. These polymers form star block, multi-branches and dendrimeric structures due to their complex molecular architectures. ${ }^{11}$

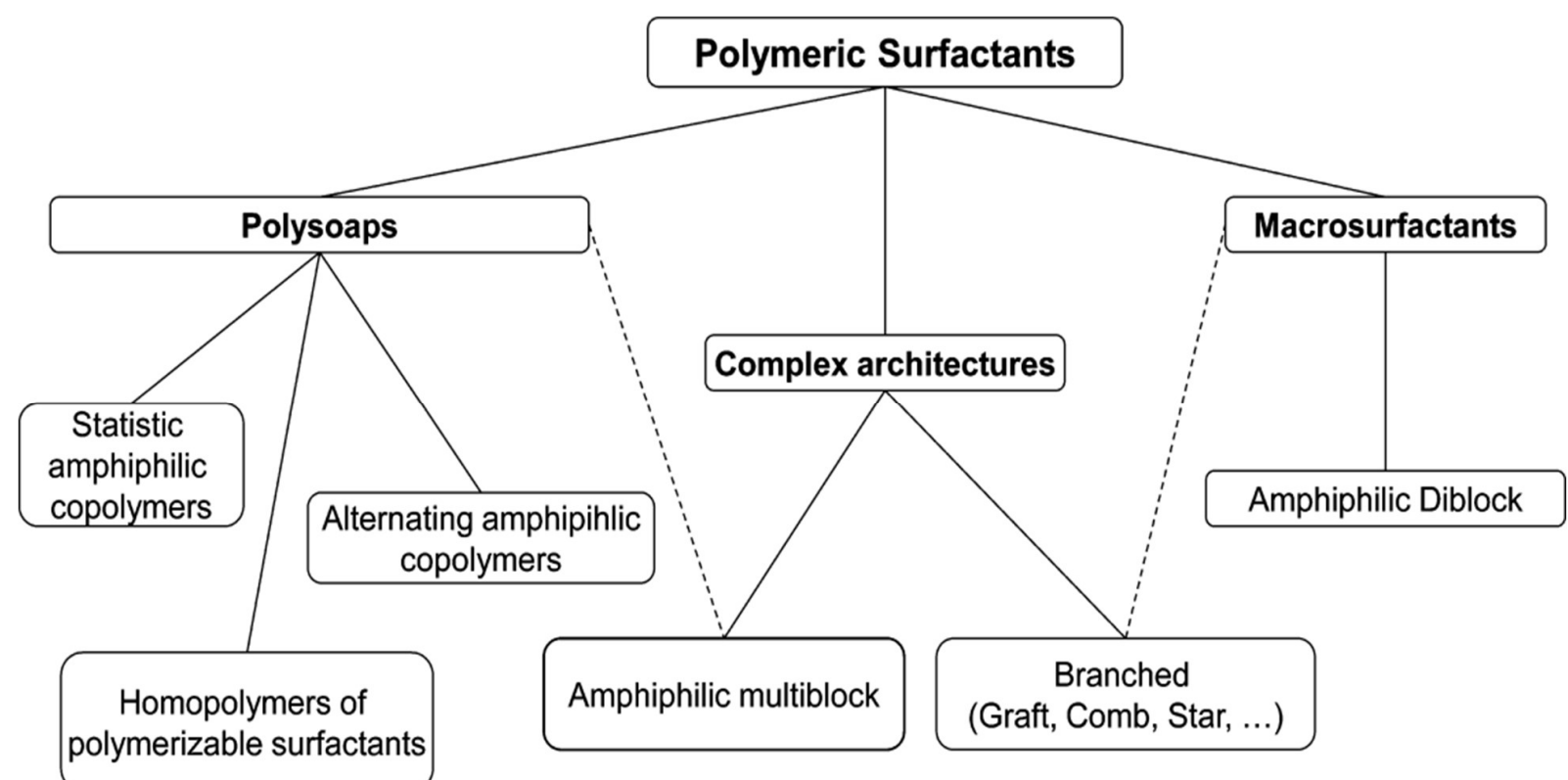

Fig.-2: Classification of Polymeric Surfactants. (Source: Raffa, Wever, Picchioni \& Broekhuis, 2015)

\section{Polysoaps}

Strauss identified polymers with aggregation properties in the 1950s and called them polysoaps. The amphipathic polysoaps possess hydrophobic and hydrophilic moieties scattered all over the backbone of the polymer. Polysoaps are composed of charged functional groups, polyelectrolytes, polyampholytes, or polyzwitterionic polymers. Among the polysoaps, the most popular ones are polyacrylamides, polyacrylates, and polysaccharides. ${ }^{12}$ The incorporation of amphiphilic monomers into the backbone of polymers during the synthesis exemplifies the micelle formation and their thermodynamics. Polysoaps possess the property of intercellular micelle formation that enhances its elasticity and surface-active character. The amphiphilic monomers are polymerized using RAFT by joining flexible hydrophilic spacer chains which is the reason behind the intramolecular self-organizational property of the polysoaps. ${ }^{13}$ Factors 
RASĀYAN J. Chem.

Vol. 13 | No. 3 |1644-1652| July - September | 2020

such as the hydrophobic tail, the length of the tail and the proportion of free micelle are monitored during the synthesis of polysoaps. These parameters will enhance or reduce the chain rigidity, electrostatic interaction and chain-chain interactions that give stable structures to the complex polysoaps. ${ }^{14}$

\section{Macrosurfactants}

This is the most functionally advanced class of polymeric surfactants. Macrosurfactnats possess a great ability to form micellar aggregates, and they exhibit straightforward stimuli-responsive behavior when subjected to aqueous solutions. These unusual properties make the application of macrosurfactants superior. The synthesis of amphiphilic copolymer macrosurfactants with good micellization and stimuli responsiveness has recently gained popularity in the detergent industry. ${ }^{15}$ Macrosurfactants possess both hydrophobic and hydrophilic moieties and demonstrate $\mathrm{pH}$ and temperature-dependent micellization. The dynamic surface activity of the macrosurfactants is also dependent on the temperature and $\mathrm{pH}$ at which the polymerization occurred. ${ }^{11}$ The hydrophobic and hydrophilic moieties of macrosurfactants possess a characteristic feature unique to them, demonstrating alternative hydrophilicity between the groups of the polymer. Macrosurfacts are thus also referred to as "schizophrenic" due to this exchangeable hydrophilicity. ${ }^{16}$

\section{Complex Polymer Architectures}

Homopolymers or heteropolymers are strategically polymerized to construct a polymer with a high degree of structural and molecular homogeneity to synthesize complex polymer architectures. These polymers are synthesized in a precise, orderly fashion while repeatedly arranging monomeric subunits that depict monodispersity. Advanced polymerization techniques applied for the synthesis of complex polymer architectures results in the formation of high order 3D structures in aqueous solutions. These complex polymeric architectures depict extremely low dispersity with stable structures and functionality. Almost all the complex polymer architectures are synthesized by anionic polymerization techniques which facilitates the formation of distinct molecular architectures such as symmetric and asymmetric star-shaped, branched or unbranched cyclic or dendritic shapes. ${ }^{17}$ These polymers depict not only complex structures but also show complex and specialized morphologies (Fig.-3).

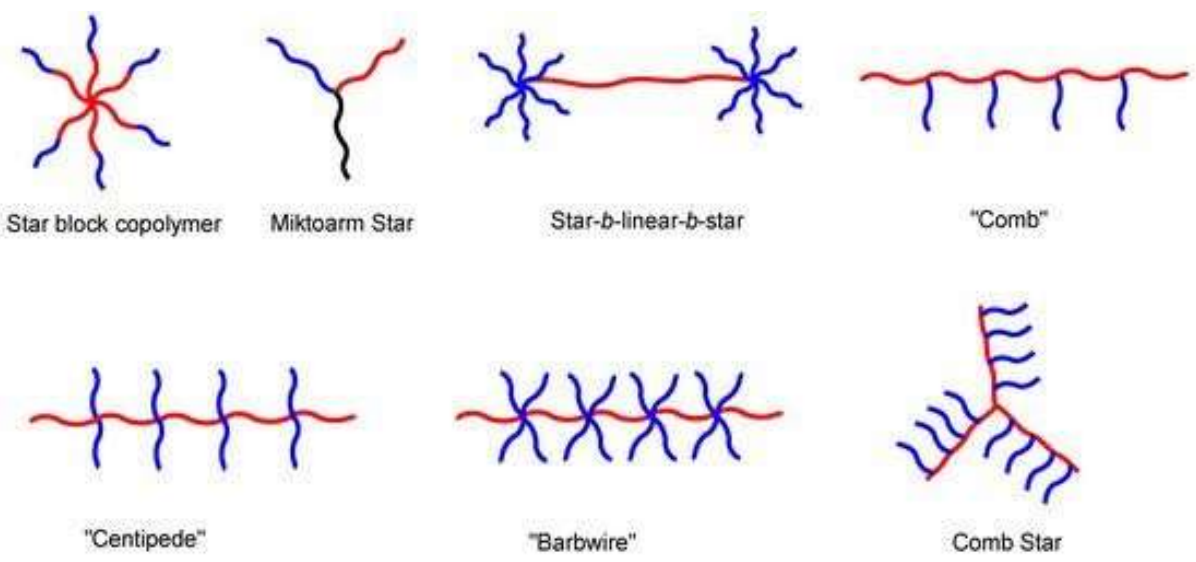

Fig.-3: Illustration of Complex Polymer Architectures(Source: Feng, Lu, Wang, Kang \& Mays, 2017)

\section{Development and Synthesis of Polymeric Surfactants}

Polymer-surfactant mixtures exhibit distinct physiochemical properties because they exist in coexisting phases. To develop and formulate functional polymeric-surfactants the critical aggregation concentrations, polymeric concentrations, polymer charge density, flexibility and hydrophobicity of the polymer chain must be assessed. The chemical behavior of the polymeric surfactants has been altered to display better desorption property and interfacial tensiometry. ${ }^{9}$ A review of the preparation of the polymeric surfactants will provide an insight into the protocols utilized in the detergent industry. 
RASĀYAN J. Chem.

Vol. 13 | No. 3 |1644-1652| July - September | 2020

\section{Formulation and Synthesis of Macro-surfactants}

The synthesis of amphiphilic polymers is dependent on the degree of protonation of carboxylic moieties and the $\mathrm{pH}$ of the solution. Associations with hydrophilic molecules to enhance their ability to form micelles in acidic conditions enables these molecules to form a hydrophobic block in water. ${ }^{18}$ The formulation of these block copolymers is not very easy because these molecules depict non-compatibility of a free carboxylic group with polymerization techniques. ${ }^{19}$ Ester moieties are utilized as monomers so that they can be easily converted into free acid after the process of polymerization has taken place. Atom transfer radical polymerization has been utilized for the formulation of amphiphilic polymer complexes. Synthesis of block copolymer linked with polyacrylic or polymethacrylic acid is one of the most popular approaches employed for the polymerization of surfactants using atom transfer radical polymerization ${ }^{20}$. These polymers demonstrate selective ionization and thus are useful for $\mathrm{pH}$-dependent micellization, where one of the two blocks selectively ionizes with a solution of different $\mathrm{pH} .{ }^{19}$ Anionic polymerization techniques were used to prepare amphiphilic copolymers, but the surfactants formulated by this method had low disparities. This property enables these polymeric surfactants to successfully remove soil particles and clean the fabric. ${ }^{10}$ The polymeric surfactants used in the detergent industry are synthesized by the solution polymerization using dimethylformamide. The copolymers are sulfonated with styrene which can be derived from decanoyl, lauroyl, and palmitoyl subchains. Precipitation reactions are used to obtain these copolymers from ether. ${ }^{3}$ Surface active macromonomers are synthesized using bromoundecyl-THP ether by organic distillation methods to formulate a slightly less pure product. Methyl poly-ethylene oxide ethers are used with organic solvents like benzene and chloroform and desiccated overnight with magnesium sulfate to procure about $85 \%$ of yield. ${ }^{21}$

\section{Synthesis of Complex Polymeric Surfactants}

Polymeric surfactants are utilized for their surface-active properties, their structurally distinct characteristics such as hyper-branched structures. Studies on the surface properties of complex polymeric surfactants have revealed that the strength of polymeric surfactants with complex poly chains can be increased by polymerization of styrene and ethylene oxide in the complex polymers. ${ }^{22}$ The availability of controlled radical polymerization methods in the preparation of amphiphilic copolymers has been achieved by incorporating combinations of ring-opening polymerizes such as styrene by using opportune multifunctional cross-linkers. ${ }^{9}$ By making the use of atomic transfer radical polymerization technique, synthesis of PMMA-PEO-PS star polymer has been successfully carried out. ${ }^{23}$ As the deactivation constant increases and the rate of propagation of styrene to $\mathrm{CuBr}$ or any other catalyst decreases, Dong et al. (2009) used only one styrene with a terminated PEO unit on available macroinitiators. This discovery of using only one streyene has brought about a positive impact of the rate of polymerization and synthesis of complex polymeric surfactants with good surface-active properties. Anionic polymerization techniques have been used to synthesize vinylic monomers with hydrophilic and hydrophobic characteristics using the atomic transfer radical polymerization technique. Gemini polymer surfactants ie. dimeric surfactants possessing two distinct hydrophilic head groups and two hydrophobic subgroups in a single molecule are also synthesized by anionic polymerization techniques. ${ }^{22}$ Recent studies have also been successful in conjugating block star polymers with dendrimeric polymers to produce long-chain, complex polymeric surfactants by atomic transfer radical polymerization of anionic moieties. Base-catalyzed ring-opening polymerization of propylene oxide (PO) has been employed to synthesize dendritic (poly (ethylene oxide) (PEO) and poly (propylene oxide) (PPO) with varying arm lengths for better surface-active properties. The PEO -PPO polymers are liked in an alternating linear fashion such as PEO-PPO-PEO- PPO for increasing the stability of the complex polymer. ${ }^{24}$ To optimize the process of synthesis of such complex polymeric surfactants Kassi et al. (2011) concluded that the nature of monomeric groups is the important prerequisites that influence the conjugation of the monomers to form complex polymers with stable structures.

\section{Polymerization and Evaluation}

The polymerization of synthetic polymeric surfactants is dependent on the characteristics of the hydrophilic and hydrophobic blocks required to be conjugated. Polystyrene, polyacrylates, polyolefin, and waterinsoluble polyethers are the most commonly used hydrophobic blocks. Negatively or positively charged 
RASĀYAN J. Chem.

Vol. 13 | No. 3 |1644-1652| July - September | 2020

monomers of vinylic or acrylic monomers are sulfonated or linked with carboxylic or amino groups or polyethylene glycol (PEG) conjugates to synthesize hydrophilic moieties. ${ }^{18}$ Atomic transfer radical polymerization (ATRP), reversible addition-fragmentation chain transfer polymerization (RAFT) and nitroxide mediated polymerization (NMP) has become the first choice of polymer chemists to synthesize amphiphilic block copolymers with small molecular weights and stable structures. ${ }^{4}$ Hydrophobically modified polyurethane (HEUR) have also been employed to develop a better understanding of aggregation patterns of polymers. ${ }^{18}$

- Atom Transfer Radical Polymerization- Polymerization by ATRP can be done by subjecting styrene sulfonate polymers to thermolysis or hydrolysis in highly basic conditions. Neopentyl ester has been recently synthesized by using ATRP to thermolysis at about $150^{\circ} \mathrm{C}$. Macromonomer of poly sodium styrene sulfonate (PSSNa) has been prepared by ATRP technique in which styrene sulphonate ethyl esters are hydrolyzed with $\mathrm{NaOH}$ or $\mathrm{KOH}$ in formamide solutions. ${ }^{12}$ Higher rates of polymerization of sodium styrene by using direct ATRP has been efficiently used to synthesize surfactant polymers of good hydrophilic-lipophilic balance. Polymerization of complex surfactants has also been facilitated by ATRP technique by conjugating it with $\mathrm{CuBr}$ catalytic system and using water or methanol mixtures as solvents. ${ }^{25}$

- Reversible Addition-Fragmentation Chain Transfer Radical Polymerization: PSSNa polymeric surfactants have been extensively prepared by RAFT polymerization techniques. RAFT has also been used to prepare PSS linked with trioctylamine (PSS-TOA) which is used as a macro-RAFT agent for the synthesis of complex polymeric surfactants that contain amphiphilic block copolymers ${ }^{19}$. Sulphonation of polystyrene after RAFT polymerization has also been used to synthesize sulfonated polystyrene block copolymers. Catalytic hydrogenation procedures have been employed to synthesize structurally stable polymeric surfactants. ${ }^{4}$

- Nitroxide-Mediated Polymerization- Polymerization of sodium styrene sulfonate with the help of tetramethylpiperidine-oxyl (TEMPO) mediated polymerization in an ethylene glycol mixture yielded polymeric surfactants that work efficiently in a homogeneous condition. Nitroxide-mediated polymerization technique using dimethylsulfoxide (DMSO) as a solvent had led to the production of styrene type copolymers that represent good hydrophilic properties. ${ }^{12}$

- Polymerization using Poly-ethylene glycol or Poly-ethylene oxide blocks: The synthesis of copolymers of PEO and PPO with varying molecular weights has been possible by open-ring anionic polymerization of ethylene oxide or condensation reaction using ethylene glycol. Amphiphilic copolymers using PEG has been used for large-scale production of hydrophobic block polymers. ${ }^{26}$ Some studies indicate that amphiphilic PEG macroinitiators are also used for the synthesis of structurally complex polymers. Thiolate precursors have also been subjected to conjugation for enhancing sequential anionic polymerization. ${ }^{18}$

- Hydrophobically modified polyurethanes: HEUR polymerization techniques have recently gained importance in the polymeric surfactants industry as these demonstrate the formation of strong copolymeric aggregates. PEO, when reacted with the diisocyanate, produces isocyanate functionalized polymer that possesses hydrophobic characteristics when reacted with aliphatic alcohols ${ }^{12}$. Such representation of superior hydrophobic copolymers is highly used in the surfactant industry. HEURs have also been synthesized by a single-step reaction involving PEO and a monoisocyanate by conjugating a hydrophobic subgroup. ${ }^{20}$

- Group Transfer Polymerization: Synthesis of acrylic acid-based polymers have been improved by the use of group transfer polymerization (GTP) techniques. Amphiphilic acrylic acid-based polymers have been synthesized by reacting acid-containing block copolymers with the precursors of polymethacrylic acid. ${ }^{27}$ Monomers with stable structures are also synthesized by GTP by using tert-butyl methacrylate (tBMA) and poly hexyl methacrylate (PHMA), followed by hydrolysis reactions. ${ }^{18}$

The quality of the complex polymeric surfactants are checked at two levels- one at the monomeric stage and one after the monomers are conjugated to form polymeric aggregates. The anionic, cationic and nonionic composition of the surfactants are analyzed by uses Ultra Performed Liquid Chromatography (UPLC), 
RASĀYAN J. Chem.

Vol. 13 | No. 3 |1644-1652| July - September | 2020

High-Performance Liquid Chromatography (HPLC) and Liquid Chromatography-Mass Spectrometry (LCMS) (10). Dynamic surface-active property and interfacial tension of the polymeric surfactants are also monitored before releasing the product for commercial use. The critical micelle concentration (CMC) of the polymeric surfactants is assessed to check the efficiency of the cleansing effect of these complex polymeric surfactants ${ }^{6}$. The ecological effect and the optimal dosage of wetting agents are also determined to evaluate the quality of the polymeric surfactants in the detergent industry. ${ }^{1}$

\section{Characterization of Polymeric Surfactants Surface and Interfacial Tension}

The surface and interfacial tension of surfactants in aqueous solutions are measured to assess the ability of the molecules of the solvent to be replaced by molecules of surfactants at the interface. The surface tension of polymeric surfactants is reduced by decreasing the length of the hydrophilic chain and increasing the length of the hydrophobic chain. ${ }^{28}$ This property shows the solubility of polymeric surfactants in aqueous solutions. By manipulating the solubility of PEO the tendency of surfactants to migrate to the interface can be controlled. ${ }^{10}$

\section{Foaming Ability}

Polymeric surfactants, when used in the detergent industry, are evaluated for their ability to foam and effectiveness as a foaming agent. Nonionic surfactants possess less foaming abilities as compared to anionic surfactants in aqueous solutions. The ability of polymeric surfactants to foam is dependent on their surface areas and the absence or presence of charged moieties. Methylation of the PEO chains, lower volumes of foam and stability of the foam can be maintained. ${ }^{3}$

\section{Emulsification}

The emulsification power of mini-emulsions or surface area-based emulsions is studied by evaluating the hydrophile-lipophilic balance (HLB). Both the hydrophobic and hydrophilic moieties conjugated on the polymers are considered for determining the extent of the emulsification of polymeric surfactants. Oil- in - water emulsion solutions are also used to investigate the strength of emulsions formed. The number of carbon atoms present in the surfactants helps in determining the optimal emulsification power necessary for polymeric surfactants to functional. ${ }^{28}$

\section{Critical Micelle Concentration (CMC)}

The surface tension method is used for determining the CMC of water-soluble triblock copolymers. The changes in the surface tension and the concentration of monomeric subunits are the attributable parameters for evaluating the $\mathrm{CMC}$ of macro surfactants and polysoaps. ${ }^{28} \mathrm{CMC}$ analysis aid in assessing the dissolution and desorption of molecules in interphase and the aqueous phase. The hydrophobic features a polymer can also be clearly understood by $\mathrm{CMC}$ analysis. ${ }^{3}$

\section{Solubilization and Other Features of Polymeric Surfactants}

Anionic polymeric surfactants conjugated with amphiphilic properties are measured by simple solubility tests conducted at various temperature gradients. The use of solubilized dyes with spectrometric analysis has demonstrated the solubility of polymers. ${ }^{10}$ The liquid-gas or liquid-liquid interface cannot be easily determined due to the large surface area of polymeric surfactants. However, the amount of molecules absorbed per unit surface area is calculated by using Gibbs adsorption equations. ${ }^{3}$ The dispersion of monomers in aqueous solutions also impacts the functions of polymeric surfactants. Spectrophotometrical analysis of the dispersibility of polymeric surfactants results in determining the dispersion rates as well as biodegradability of the amphiphilic polymeric surfactants. ${ }^{28}$

\section{Application of Polymeric Surfactants}

Polymeric surfactants have applications in the cosmetics, textile $\&$ dyeing and pharmaceuticals along with application in detergent industries. The CMC levels and the amount of hydrocarbons molecules present increase the efficiency of these surfactants. They possess specialized features that reduce the surface tension 
RASĀYAN J. Chem.

Vol. 13 | No. 3 |1644-1652| July - September | 2020

of water and the interfacial tension at the oil-water surfaces. The polymeric surfactants display a wide range of properties such as elevated surface activity, low CMC and extraordinary rheology with self-assimilation features demonstrates their potential applications as detergents and cleaning agents. Anionic and cationic polymers are used as amicable personal hygiene products that prevent skin or eye irritation. These surfactants also possess antimicrobial properties with lasting effects. ${ }^{29}$ The low CMC of the polymeric surfactants reduces their irritancy and toxicity levels, whereas increases its cleansing properties. As the hydrophobic chains are tightly packed at interfaces, cohesive interfacial films depict superior emulsifying and dispersing properties. This tight packing of these polymers also enhances their foaming characteristics. Polymers with short and branched linked chain conjugated in the backbone possess dynamic wetting properties. Superior solubility levels to solubilize water-insoluble materials in the cleaning process aids in the efficient removal of stains, paints, inks, and dye from both synthetic and natural fabrics. Sulfonates polymeric surfactants offer great solubility with good foaming behavior and stability in acidic and alkaline solutions. Such surfactants are widely known and used for their ability to bleach fabrics at higher as well as lower temperatures. Excellent surface-active properties of complex polymeric surfactants enable commercial use in textile processing units to improve the quality of dyeing and printing. ${ }^{30}$

\section{CONCLUSION}

The application of polymeric surfactants in the detergent industry has uplifted the face of detergents used for cleaning purposes because of the unique features. Polymeric surfactants synthesized by conjugating hydrophilic and hydrophobic moieties represent a new class of advanced surfactants. These surfactants display versatility and at times resemble their detergent counterparts like both behave as surfactants when exposed to lower interfacial tension. The polymeric features that surfactants possess give them additional structural stability. One of the best features of polymeric surfactants is that they can be fine-tuned to display higher-order aggregation behaviors that enhance their surface activity features. The specific nature of the polymeric surfactants is their ability to form strong polymer-surfactant aggregates in bulk. Some aggregates are even formed at low ph. This is due to the electrostatic interactions that exist between the monomers. This feature of the polymeric surfactants intensifies their micellization property and enhances the cleaning effect. Copolymerization techniques like RAFT and ATRP ensure that polymers are formed as a result of stable bonding and linkages. Intermolecular and intramolecular adjustments can be made to enhance the molecular aggregation of monomers and gives rise to stable complex structures with varying structures. The ability to form intermolecular aggregates and the flexibility to fine-tune these structures is the most attractive feature of polymeric surfactants. This ability has also made amphiphilic copolymers to be available in various detergent and cleaning industries. All types of polymeric surfactants possess the characteristic feature of self-assembly which has increased their potential application in various industries. As the polymerization of polymeric surfactants can be controlled and manipulated to suit the requirements of the industry, it has gained popularity in industries apart from the detergent industry as well.

\section{REFERENCES}

1. M. S. Kamal, I. A. Hussein, A. S. Sultan, Energy \& Fuels, 31(8), 7701(2017), DOI: 10.1021/acs.energyfuels.7b00353

2. P. Raffa, A.A. Broekhuis, F. Picchioni, Journal of Petroleum Science and Engineering, 145, 723(2016), DOI: $10.1016 /$ j.petrol.2016.07.007

3. M.Z. Mohamed, D.A. Ismail, A.S. Mohamed, Journal of Surfactants and Detergents, 8(2), 175 (2005), DOI: $10.1007 / \mathrm{s} 11743-005-344-4$

4. E. Guzmán, S. Llamas, A. Maestro, L. Fernández-Peña, A. Akanno, R. Miller, R.G. Rubio, Advances in Colloid and Interface Science, 233, 38(2016), DOI:10.1016/j.cis.2015.11.001

5. C.D. Bain, P.M. Claesson, D. Langevin, R. Meszaros, T. Nylander, C. Stubenrauch, R. Von Klitzing, Advances in Colloid and Interface Science, 155(1-2), 32(2010), DOI:10.1016/j.cis.2010.01.007

6. S. Llamas, E Guzman, F. Ortega, N. Baghdadli, C. Cazeneuve, R.G. Rubio, G.S. Luengo, Advances in Colloid and Interface Science, 222, 461(2015), DOI:10.1016/j.cis.2014.05.007

7. Z.L. Wan, L.Y. Wang, J.M. Wang, Q. Zhou, Y. Yuan, Q.X. Yang, Food Hydrocolloids, 39, 127(2014), DOI:10.1016/j.foodhyd.2014.01.007 
RASĀYAN J. Chem.

Vol. 13 | No. 3 |1644-1652| July - September | 2020

8. K. Szczepanowicz, U. Bazylińska, J. Pietkiewicz, L. Szyk-Warszyńska, A.K. Wilk, P. Warszyński, Advances in Colloid and Interface Science, 222,678(2015), DOI:10.1016/j.cis.2014.10.005

9. M. Showell,(2016), Handbook of Detergents, Part D: Formulation. CRC Press.

10. P. Raffa, D.A.Z. Wever, F. Picchioni, A.A. Broekhuis, Chemical Reviews, 115(16), 8504(2015), DOI: $10.1021 / \mathrm{cr} 500129 \mathrm{~h}$

11. B. Kronberg, K. Holmberg \& B. Lindman, (2014), Surface Chemistry of Surfactants and Polymers, John Wiley \& Sons.

12. Y. Li. X. Zhao, S. Li. W. Wang, X. Wang, S. Chen, X. Xie, International Journal of Hydrogen Energy, 42(50), 30013(2017), DOI:10.1016/j.ijhydene.2017.08.167

13. G. Polymeropoulos, G. Zapsas, K. Ntetsikas, P. Bilalis, Y. Gnanou, N. Hadjichristidis, Macromolecules, 50(4), 1253(2017), DOI:10.1021/acs.macromol.6b02569

14. W.M. Wan, P.D. Pickett, D.A. Savin, C.L. McCormick, Polymer Chemistry, 5(3), 819(2014), DOI: $10.1039 / \mathrm{c} 3 p y 01073 b$

15. W. Moussa, O. Colombani, L. Benyahia, T. Nicolai, C. Chassenieux, Polymer Bulletin, 73(10), 2689(2016), DOI:10.1007/s00289-016-1615-5

16. A. Durand, E. Marie, Advances in Colloid and Interface Science, 150(2), 90(2009), DOI:10.1016/j.cis.2009.07.002

17. A.E.Smith, X. Xu, S.E. Kirkland-York, D.A. Savin, C.L. McCormick, Macromolecules, 43(3), 1210(2010), DOI: $10.1021 / \mathrm{ma902378k}$

18. C.A. Figg, R.N. Carmean, K.C. Bentz, S. Mukherjee, D.A. Savin, B.S. Sumerlin, Macromolecules, 50(3), 935 (2017), DOI:10.1021/acs.macromol.6b02754

19. I. Chaduc, W. Zhang, J. Rieger, M. Lansalot, F. D'Agosto, B. Charleux, Macromolecular Rapid Communications, 32(16), 1270(2011), DOI:10.1002/marc.201100240

20. A. Khan and M. Siddiq, Journal of Applied Polymer Science, 118(6), 3324(2010), DOI: 10.1002/app.32226

21. J. Liu, C.H. Chew, L.M. Gan, Journal of Macromolecular Science, Part A: Pure and Applied Chemistry, 33(3), 337(1996), DOI:10.1080/10601329608014324

22. E. Kassi, E. Loizou, L. Porcar, C.S. Patrickios, European Polymer Journal, 47(4), 816(2011), DOI: $10.1016 /$ j.eurpolymj.2010.09.011

23. Y.Q. Dong, B.T. Dong, F.S. Du, J.Q. Meng, Z.C. Li, Polymer, 50(1), 125(2009), DOI: $10.1016 /$ j.polymer.2008.10.046

24. D.A.Z. Wever, E. Riemsma, F. Picchioni, A.A. Broekhuis, Polymer, 54(21), 5456(2013), DOI: 10.1016/j.polymer.2013.07.039

25. K. Matyjaszewski, Macromolecules, 45(10), 4015(2012), DOI:10.1021/ma3001719

26. Q. Cui, F. Wu, E. Wang, Polymer, 52(8), 1755(2011), DOI:10.1016/j.polymer.2011.02.022

27. S.P. Rannard, N.C. Billingham, S.P. Armes, J. Mykytiuk, European Polymer Journal, 29(2-3), 407(1993), DOI:10.1016/0014-3057(93)90112-S

28. A. El Menam Eissa, M. El Hefnawy, S.Bader, M. Radwan, M. Deef Allah, Journal of Surfactants and Detergents, 15(4), 411(2012), DOI:10.1007/s11743-011-1324-x

29. N. Kumar, R. Tyagi, Journal of Dispersion Science and Technology, 35(2), 205(2014), DOI: $10.1080 / 01932691.2013 .780243$

30. A. Bureiko, A. Trybala, N. Kovalchuk, V. Starov, Advances in Colloid and Interface Science, 222, 670(2015), DOI:10.1016/j.cis.2014.10.001

[RJC-5387/2020] 